\title{
Influence of auto-organization and fluctuation effects on the kinetics of a monomer-monomer catalytic
}

\author{
scheme. \\ P.Argyrakis ${ }^{1}$, S.F.Burlatsky ${ }^{2}$, E.Clément ${ }^{3}$, and G.Oshanin ${ }^{4}$ \\ 1 Department of Physics, University of Thessaloniki, 54006 Thessaloniki, Greece \\ ${ }^{2}$ LSR Technologies, Inc., 898 Main St, Acton, MA 01720-5808 USA \\ ${ }^{3}$ Laboratoire AOMC, Université Paris VI, 4 Place Jussieu, 75252 Paris Cedex 05, France \\ ${ }^{4}$ Laboratoire de Physique Théorique des Liquides, Université Pierre et Marie Curie, 4 place \\ Jussieu, 75252 Paris Cedex 05, France
}

\begin{abstract}
We study analytically kinetics of an elementary bimolecular reaction scheme of the LangmuirHinshelwood type taking place on a $d$-dimensional catalytic substrate. We propose a general approach which takes into account explicitly the influence of spatial correlations on the time evolution of particles mean densities and allows for the analytical analysis. In terms of this approach we recover some of known results concerning the time evolution of particles mean densities and establish several new ones.
\end{abstract}

PACS No: 82.30.Vy, 82.20.-w, 05.40

\section{INTRODUCTION}

Catalytically activated processes play a significant role in numerous technologies as they serve to produce required products from the species which are non-reactive in normal physical conditions; these chemically stable species may, however, enter into reaction in the presence of some third substance - the catalytic substrate [1 [3]. Despite of the widespread use of such processes, the knowledge of the underlying physics and chemistry still rests largely on phenomenological ideas and prescriptions, and thus remains a challenge for fundamental research [1]. 
At the simplest, mean-field level of description, reaction kinetics theory presumes that the reaction rate should be considered as the product of the reactant densities and the rate constant, which is dependent on the nature of the binding forces and on the particles' dynamics. This rate constant is proportional to the hopping rate if the process is diffusion controlled, or to the reaction frequency, in case when the reaction is kinetically controlled [6 9]. Hence, a clear cut separation is presumed to exist between the local variables, that could be derived, say, from quantum mechanics, and collective variables, expressed in the most simple way as the product of mean densities of the particles involved.

Recently, one of the most active developments in the field has been the recognition of the substantial importance of the multi-particle effects, spatial fluctuations and selforganization, as opposed to conventional local energetic considerations, which emphasized the purely chemical constraints and focused on the refined descriptions of the elementary reaction act. Subsequently, statistical physics concepts were introduced in order to describe anomalous fluctuation-induced behaviors of non-catalytic chemical reactions [10 25] and simplest catalytic schemes [26 36], as well as such to gain a better understanding of such collective phenomena as wave formation, presence of excitable media or stochastic aggregation in chemical systems (see Refs. [28,32,33] and references therein). One conclusion that can be drawn from the statistical physics approach is that fluctuations, either spatial or temporal, may drive the reactive system into a set of new states, that cannot be understood and described in terms of mean-field kinetic equations.

In this paper we discuss in detail the influence of spatial fluctuations, statistical selforganization effects and effects of random diffusive motion of reactants on the kinetics of catalytic reactions, using as a particular example the Langmuir-Hinshelwood type reaction scheme. This reaction process, which is also often referred to as the monomer-monomer catalytic scheme, involves two different kinds of species, $A$ and $B$, which are deposited (continuously in time with mean intensity $I$ ) onto the catalytic substrate by an external source, diffuse on the substrate and react at encounters forming an inert reaction product $O, A+B \rightarrow O$, which is then removed from the system. Our aims here are twofold. First, 
we show that this seemingly simple catalytic reaction (which has, in fact, several practical applications (see, e.g. Ref. [26,27])) shows quite a rich behavior and represents an ideal illustrative example of the statistical physics effects in the reaction kinetics, which may be generic to more complicated schemes involved in real "stuff" catalytic processes. Second, we develop a unified analytical description which takes into account explicitly the influence of spatial correlations, dimensionality of space and the way how the particles are introduced into the reaction bath on the time evolution of observables - mean particle densities. Moreover, our approach can be routinely generalized for kinetic description of more complex reaction schemes.

We focus here on several different aspects of the Langmuir-Hinshelwood reaction scheme. In particular, we address the question of how the kinetics depends on the dimensionality $d$ of the reactive system. In addition to the standard Langmuir-Hinshelwood model in which the catalytic substrate is a two dimensional flat surface, we analyse kinetics of $A+B \rightarrow 0$ reactions followed by an external input of reactive species in one- and three-dimensional systems, i.e. the situations appropriate to reactions in capillary geometries [37] and annealing of the radiation damage in solids [14]. We show that the monomer-monomer catalytic reaction proceeds quite differently in low dimensional $(d=1,2)$ and three-dimensional systems. Besides, we examine how the way of particles' injection into the system affects the properties of stationary states and also how these stationary states are approached in time. We consider here two different types of external input; in the first one (case I) the $A$ and $B$ particles are introduced independently of each other at random moments of time and at random positions in space, while in the second case (case II) an $A$ and a $B$ species are introduced in correlated pairs of a fixed radius, the pairs being injected at random moments of time and at random positions in space. We show that also the way of input does matter significantly and may result in a completely different behavior.

The paper is structured as follows: In Section II we formulate the model, introduce a general analytic approach and, in terms of this approach, derive closed-form equations describing the time evolution of mean particles' densities and pairwise correlation functions. 
In Section III we present solution to these equations in one-, two- and three-dimensional systems corresponding to different ways of particles injection to the reaction bath. Finally, we conclude in Section IV with a brief summary of our results and discussion.

\section{DEFINITIONS AND BASIC EQUATIONS.}

In this section we present kinetic description of the monomer-monomer catalytic scheme involving diffusive particles in terms of a certain analytical approach, which takes explicitly into account the influence of pairwise correlations on the time evolution of mean densities; such a description has been first proposed in Ref. [11], which analysed the effects of fluctuations on the kinetics of $A+B \rightarrow O$ reactions and yielded the celebrated $t^{-d / 4}$-law for the decrease of particles mean densities. The subsequent works [22,24,25, 38 46] extended this approach to more general reaction schemes (e.g. reversible and coagulation reactions), three-body and catalytic reactions, and also included the possibility of mutual long-ranged interparticle interactions. Here we will focus on the application of this approach to the analytical description of the $A+B \rightarrow O$ reactions kinetics in systems with a continuous, random external input of the reactive species. We will recover some of already known results obtained for the monomer-monomer catalytic scheme involving diffusive monomers and will establish several new ones concerning the long-time relaxation of mean particles density to their steady-state values and the dependence of this steady-state densities on the system parameters.

We start with the formulation of the model. Consider a $d$-dimensional reaction bath of volume $V$ (we suppose that $V$ is sufficiently large such that we can discard different finite-size effects, e.g. hard-core exclusion between particles, the termination of reaction within a finite time interval or saturation (poisoning)) in which $A$ and $B$ particles are continiously introduced by an external random source. The statistical properties of the source will be defined below. After injection to the system, the $A$ and $B$ particles begin to diffuse. For simplicitly we assume that their diffusion constants are equal to each other, 
i.e. $D_{A}=D_{B}=D$. It will be made clear below that such a description is also appropriate to the case of non-equal diffusivities; the calculations in this case will be only essentially more lengthy [47]. Now, the reaction event is defined as follows: When any two $A$ and $B$ particles approach each other at a fixed separation $R$ (the reaction radius), they may enter into reaction forming (an inert with respect to the reaction) reaction product $O$. The recombination upon an encounter of $A$ and $B$ happens with a finite probability $p$ (with probability $q=1-p$ the particles can be reflected) which defines the constant of an elementary reaction act, $K$. This constant describes the intrinsic chemical activities of $A$ and $B$ molecules and is dependent on the nature of the intra- and intermolecular binding forces. In the following we will suppose that this purely "chemical" constant is known a priori. Besides, we will assume that $K$ is large (i.e. the probability of particles reflection from each other is low) and thus will emphasize the "statistical physics" effects on the reaction kinetics, rather than the effects of chemical constraints. Consequently, all the factors which are exponentially small with $K$ will be neglected here.

Now, we define the statistical properties of the particles generation more precisely. Let $I_{A}(r, t)$ and $I_{B}(r, t)$ be the local, at the point with the radius-vector $r$, intensities of the production rates of $A$ and $B$ particles. We take that the volume average values of the production rates obey

$$
\frac{1}{V} \int_{V} d r I_{A}(r, t)=\frac{1}{V} \int_{V} d r I_{B}(r, t)=I,
$$

which means that particles generation is steady in time and the mean production rates $I$ of $A$ and $B$ particles are equal to each other.

Defining the correlations in the production rates $I_{A, B}(r, t)$ we will consider here two different situations. In the first one (case I), we suppose that $A$ and $B$ particles are introduced into the reaction bath statistically independent of each other [16 22]; that is, the fluctuations of the sources are correlated neither in space, nor in time. In this case, we have

$$
\frac{1}{V} \int_{V} d r I_{A}(r, t) I_{A}(r+\lambda, t+\tau)-I^{2}=I \delta(\lambda) \delta(\tau),
$$




$$
\begin{aligned}
& \frac{1}{V} \int_{V} d r I_{B}(r, t) I_{B}(r+\lambda, t+\tau)-I^{2}=I \delta(\lambda) \delta(\tau) \\
& \frac{1}{V} \int_{V} d r I_{B}(r, t) I_{A}(r+\lambda, t+\tau)=0 \quad(c)
\end{aligned}
$$

In the second case we suppose that $A$ and $B$ particles are introduced as correlated $A-B$ pairst, separated by a fixed distance $\lambda_{g}[17,20$ 22]. Such type of external generation may arise in chemical systems in which complex reaction product $O$ is continuously forced to breakup by an external radiation (say, laser pulses) into the correlated pairs of the component molecules. Here the radius of pair $\lambda_{g}$ will be mainly determined by the difference of energy "attributed" to $O$ and the energy required to dissociate the reaction product. Another example in which such pairs are produced is the annealing of radiation damage in solids. When the solid is irradiated, atoms are knocked out of their places in the lattice to become interstitials and leave behind a vacancy, and then the vacancies and interstitials diffuse and recombine. In case II, different $A-B$ pairs are statistically uncorrelated and occur, with an average intensity $I$, at random positions in the reaction bath. Then, the fluctuations of the sources obey [17,39]:

$$
\begin{aligned}
& \frac{1}{V} \int_{V} d r I_{A}(r, t) I_{A}(r+\lambda, t+\tau)-I^{2}=I \delta(\lambda) \delta(\tau), \quad(a) \\
& \frac{1}{V} \int_{V} d r I_{B}(r, t) I_{B}(r+\lambda, t+\tau)-I^{2}=I \delta(\lambda) \delta(\tau), \quad(b) \\
& \frac{1}{V} \int_{V} d r I_{B}(r, t) I_{A}(r+\lambda, t+\tau)=\gamma_{d}\left(\lambda_{g}\right) I \delta\left(|\lambda|-\lambda_{g}\right) \quad(c)
\end{aligned}
$$

In Eq.(3.c), the parameter $\gamma_{d}\left(\lambda_{g}\right)$ is the normalization factor which arises because of different possible angular orientations of a given $A-B$ pair in a $d$-dimensional continuum; the value of $\gamma_{d}\left(\lambda_{g}\right)$ depends on the dimensionality of the reaction bath and for $d=1,2$ and 3 equals respectively $1,\left(2 \pi \lambda_{g}\right)^{-1}$ and $\left(4 \pi \lambda_{g}^{2}\right)^{-1}$.

Let $C_{A}(r, t)$ and $C_{B}(r, t)$ denote the local densities of $A$ and $B$ particles at point with radius-vector $r$ at time $t$. The time evolution of local densities due to the diffusion of species,

\footnotetext{
${ }^{1}$ The particles in the pair can, of course, diffuse apart after injection.
} 
their reaction and an external production can be described by the following reaction-diffusion equations [16, 19, 20]:

$$
\begin{aligned}
\dot{C}_{A}(r, t)= & -\gamma_{d}(R) K \int_{V} d r^{\prime} \delta\left(\left|r-r^{\prime}\right|-R\right) C_{A}(r, t) C_{B}\left(r^{\prime}, t\right)+ \\
& +D \Delta_{r} C_{A}(r, t)+I_{A}(r, t) \\
\dot{C}_{B}(r, t)= & -\gamma_{d}(R) K \int_{V} d r^{\prime} \delta\left(\left|r-r^{\prime}\right|-R\right) C_{B}(r, t) C_{A}\left(r^{\prime}, t\right)+ \\
& +D \Delta_{r} C_{B}(r, t)+I_{B}(r, t),
\end{aligned}
$$

where the symbol $\Delta_{r}$ denotes the $d$-dimensional Laplace operator acting on the spatial variable $r$, and the integration with the delta-function $\delta\left(\left|r-r^{\prime}\right|-R\right)$ accounts for all possible orientations of an $A-B$ pair, at which an elementary reaction act can take place.

Now, an experimentally accessible property is not, however, the local density, but rather its volume averaged value

$$
C(t)=\frac{1}{V} \int_{V} d r C_{A, B}(r, t)
$$

To find an equation which governs the time evolution of $C(t)$, let us first represent the local densities in the form

$$
C_{A, B}(r, t)=C(t)+\delta C_{A, B}(r, t)
$$

where $\delta C_{A, B}(r, t)$ will denote local deviations of particles' densities from their mean values. By definition,

$$
\frac{1}{V} \int_{V} d r \delta C_{A, B}(r, t)=0
$$

Then, substituting Eq.(7) into Eqs.(4) and (5) and taking the volume average, we get the following equation

$$
\dot{C}(t)=-K\left[C^{2}(t)+G_{A B}(|\lambda|=R, t)\right]+I,
$$

in which $G_{A B}(\lambda, t)$ stands for the pairwise, central correlation function of the form 


$$
G_{A B}(\lambda, t)=\frac{1}{V} \int_{V} \int_{V} d r d r^{\prime} \delta\left(r-r^{\prime}-\lambda\right) \delta C_{A}(r, t) \delta C_{B}\left(r^{\prime}, t\right)
$$

the variable $\lambda$ being a $d$-dimensional correlation parameter.

Therefore, Eq.(9) shows that the time evolution of the mean particle density is ostensibly coupled to the evolution of the pairwise correlations in the reactive system. Neglecting these correlations, i.e. setting $G_{A B}(\lambda, t)=0$, which is equivalent to the traditional, "mean-field" assumption that the particles' spatial distribution is uniform, we obtain the customary, textbook "law of mass action". Such an approximation predicts a linear in time growth of mean density at relatively short times, i.e.

$$
C(t) \propto I t
$$

and, in the large- $t$ limit, an exponentially fast relaxation to the equilibrium density $C(t=$ $\infty)=(I / K)^{1 / 2}$, i.e.

$$
C(t) \propto\left(\frac{I}{K}\right)^{1 / 2}\left[1-\exp \left(-2(I K)^{1 / 2} t\right)+\ldots\right]
$$

The short-time behavior as in Eq.(11) is quite reasonable and describes the regime in which the particles are merely added into the (initially empty) system by the external source and the reaction between them is negligible, i.e. the regime in which particle density remains very small. As for the analytical prediction in Eq.(12), one may question its validity on intuitive grounds. First, in the system under consideration diffusive motion of particle is the only mechanism to bring particles together and let them react. This motion is essentially $d$-dependent and should evidently entail $d$-dimensional behaviors, whilst Eq.(12) is independent of the dimensionality of the reaction bath. Second, it shows that with an increase of the chemical reaction constant $K$ the equilibrium density tends to zero, which is apparently an artificial behavior. Below we will show that the actual behavior of $C(t)$ as $t \rightarrow \infty$ is very different. This turns out to depend essentially on the dimensionality of the reactive system and also on the way how the particles are injected into the system. We show analytically that in the case I, when $A$ and $B$ particles are introduced into the system 
statistically independ of each other, in low dimensional systems (i.e. $d=1,2$ ) there occurs a strong reaction-induced spatial organization of particles. Here the absolute value of the pairwise correlation function $G_{A B}(R, t)$ (which is negative) grows in time, which induces an unlimited (in absence of hard-core exclusion) growth of mean particles' densities $C(t)$. Therefore, in the case I in low dimensional systems the steady state does not exist, in a striking contradiction to the prediction of Eq.(12). Similar results have been obtained previously in Refs. [16 22,39]. In the case I, in three dimensional systems the steady state density is well-defined but, however, turns out to be different from that predicted by Eq.(12). In particular, it is dependent on particles' diffusion constant, which reflects the pathological behavior of correlations in the reactive system [16,17,22, 39]. Actually, we show that correlations of fluctuations in particles local densities are essentially long-ranged and obey, in the steady-state, an algebraic law $G_{A B}(|\lambda|, t) \sim 1 /|\lambda|$. Finally, we proceed to show that in three dimensional systems with uncorrelated generation of particles the long-time relaxation of mean particles density to its steady-state value is described by a power-law with characteristic exponent $-1 / 2$, in contrast to the exponential dependence predicted by Eq.(12). Such a behavior has been first conjectured in Ref. [41] and here will be deduced analytically. For the case II, we show that steady-state density exists in all dimensions [17,22, 39]. However, its value is also different from that predicted by Eq.(12) and depends, for instance, on the radius of the generated pairs, $\lambda_{g}$. The approach to the steady state in this case is also not exponential in time and is described by a universal algebraic law, reminiscent of the long-time approach to the equilibrium in reversible chemical reactions [12,13, 38,42 .

We turn back to Eq.(9) and continue our analysis of the binary reaction kinetics taking into account the influence of pairwise correlations on the time evolution of $C(t)$. Omitting the details of the derivation, which can be found in Refs. [39,40, we write down the system of equations for the time evolution of the pairwise correlation functions

$$
\begin{aligned}
\dot{G}_{A B}(\lambda, t)= & -K C(t)\left[2 G_{A B}(\lambda, t)+G_{A A}(\lambda, t)+G_{B B}(\lambda, t)\right]+ \\
& +2 D \Delta_{\lambda} G_{A B}(\lambda, t)+I_{A B}(\lambda)+T_{A B}(\lambda)
\end{aligned}
$$




$$
\begin{aligned}
\dot{G}_{A A}(\lambda, t)= & -2 K C(t)\left[G_{A B}(\lambda, t)+G_{A A}(\lambda, t)\right]+ \\
& +2 D \Delta_{\lambda} G_{A A}(\lambda, t)+I \delta(\lambda)+T_{A A}(\lambda), \\
\dot{G}_{B B}(\lambda, t)= & -2 K C(t)\left[G_{A B}(\lambda, t)+G_{B B}(\lambda, t)\right]+ \\
& +2 D \Delta_{\lambda} G_{B B}(\lambda, t)+I \delta(\lambda)+T_{B B}(\lambda),
\end{aligned}
$$

where $\Delta_{\lambda}$ denotes the Laplace operator acting on the $d$-dimensional variable $\lambda$, the symbol $I_{A B}(\lambda)$ in Eq.(13) describes the correlations in the production rates of $A$ and $B$ particles; it is equal to zero in the case I (uncorrelated generation of particles) and to

$$
I_{A B}(\lambda)=\gamma_{d}\left(\lambda_{g}\right) I \delta\left(|\lambda|-\lambda_{g}\right)
$$

in case II, when particles are introduced into the system in correlated $A-B$ pairs. Finally, in Eqs.(13) to (15) the terms $T_{i j}$ denote the correlation functions of the third order.

The time evolution of the pairwise correlations is coupled to the evolution of the thirdorder correlations, which, in turn, depends on the correlations of the fourth order. Thus the non-linearity of the reaction-diffusion Eqs.(4) and (5) entails an infininite hierarchy of equations for correlation functions and one has to resort to some approximate methods.

The most commonly used method invokes truncation of this hierarchy approximating the third-order correlation functions in terms of $C(t)$ and $G_{i j}(\lambda, t)$ [50]. Such an approach, as it was first noticed in Ref. [14], results in the Smoluchowski-type approximate results with improved numerical coefficients and is appropriate for the description of the single-species reactions $A+A \rightarrow O$, but not for the description of reactions involving two different types of particles. The point is that such an approximation misses an important conservation law, which is specific for $A+B \rightarrow O$ reactions. Namely, the reaction process conserves the difference $Z(r, t)$ of local densities, $Z(r, t)=C_{A}(r, t)-C_{B}(r, t)$, which changes only due to the diffusion of particles and thus is a pure diffusive mode of the system. Conservation of $Z(r, t)$ entails, in turn, the conservation of the combination of pairwise correlation functions, 
$S_{-}(\lambda, t)=G_{A A}(\lambda, t)+G_{B B}(\lambda, t)-2 G_{A B}(\lambda, t)$, which is also a pure diffusive modef Consequently, only such truncation scheme should correctly describe the behavior of the binary reaction, which does not violate the important conservation laws [14].

The most simple truncation scheme, which preserves the conservation laws, has been first proposed in Ref. [11]. In this scheme the third-order correlation functions, i.e. $T_{i j}$, were set equal to zero. Such a truncation, as it was shown in Refs. [22] and [38 40] is equivalent to the assumption that fields $\delta C_{A, B}(\lambda, t)$ have Gaussian distribution. Then, the fourthorder correlation functions automatically decouple into the product of pairwise correlation functions and the third-order correlations are equal to zero. Such an approach leads to, for instance, the correct long-time decay law of the particles mean densities, i.e. the $t^{-d / 4}$-law, but fails to reproduce correctly the intermediate time behaviors; at intermediate times this approach predicts essentially the same behavior as the formal kinetic "law of mass action" and thus disregards the effects of particles' diffusion at the intermediate-time stage.

This shortcomming has been revisited and improved in Refs. [38 40], in which it has been shown that correlation functions of the third order are small only in the limit $|\lambda|>R$, while in the domain $|\lambda| \approx R$ they are singular and this singularity has an impact on the behavior of the pairwise correlation at the intermediate times. In a discrete-space picture, essential at scales $|\lambda| \approx R$, the third-order correlation functions have been computed explicitly [39],

$$
T_{A A} \approx T_{B B} \approx T_{A B} \approx \gamma_{d}(R) \dot{C}(t) \delta(|\lambda|-R)
$$

Substituting Eq.(17) into Eqs.(13) to (15) one gets then a closed with respect to $C(t)$ and $G_{i j}(\lambda, t)$ system of equations. To solve them, it is expedient to represent the pairwise correlations in the form

$$
G_{i j}(\lambda, t)=\hat{G}_{i j}(\lambda, t)+g_{i j}(\lambda, t)
$$

where $\hat{G}_{i j}(\lambda, t)$ denotes a "singular" part of the pairwise correlation functions, which accounts merely for the behavior of the third-order correlations, and $g_{i j}(\lambda, t)$ - the "fluc-

\footnotetext{
${ }^{2}$ One may readily verify [39] that $T_{A A}+T_{B B}-2 T_{A B}$ is exactly equal to zero.
} 
tuational" part, which accounts for the fluctuation spectrum of the external source and fluctuations stemming out of reaction and diffusive processes.

The "singular" part of the pairwise correlation functions has been determined in Ref. [39]. In particular, the leading at sufficiently large times behavior of $\hat{G}_{i j}(|\lambda|=R, t)$ have been found to be as follows:

$$
\hat{G}_{i j}(R, t) \approx \dot{C}(t)(\pi t / 8 D)^{1 / 2}
$$

in one-dimensional,

$$
\hat{G}_{i j}(R, t) \approx \dot{C}(t) \frac{\ln \left(D t / R^{2}\right)}{8 \pi D}
$$

and

$$
\hat{G}_{i j}(R, t) \approx \dot{C}(t) 8 \pi D R
$$

in two-, and three-dimensional systems, respectively.

Now, inserting Eqs.(19) to (21) to Eq.(9) we obtain the following equation for the time evolution of particles mean density

$$
\dot{C}(t)=-\frac{K K_{S}(d)}{K+K_{S}(d)}\left[C^{2}(t)+g_{A B}(|\lambda|=R, t)\right]+\frac{I}{1+K / K_{S}(d)}
$$

where $K_{S}(d)$ obeys, as $t \rightarrow \infty$,

$$
\begin{gathered}
K_{S}(d=1) \approx\left(\frac{8 D}{\pi t}\right)^{1 / 2}, \\
K_{S}(d=2) \approx \frac{8 \pi D}{\ln \left(D t / R^{2}\right)},
\end{gathered}
$$

and

$$
K_{S}(d=3) \approx 8 \pi D R
$$

One may readily notice that in three-dimensions the $K_{S}(d)$, Eq.(25), coincides exactly with the so-called "diffusive" Smoluchowski constant; a reaction constant which has been 
first calculated by Smoluchowski [6] in his approximate description of the effects of diffusion on the chemical reactions kinetics. This constant accounts for, heuristically, the "resistivity" of random, diffusive transport of particles with respect to reaction. Employing the Smoluchowski method, the analogues of such a constant have been obtained in Ref. 449 for oneand two-dimensional systems. Remarkably, our results in Eqs.(23) and (24) coincide with those obtained in Refs. [9] and [49]. We note also that the prefactor before the brackets in Eq.(22), i.e. the ratio $K_{a p p}=K K_{S}(d) /\left(K+K_{S}(d)\right)$, is the so-called effective or apparent reaction constant, which was first derived for three-dimensional systems in Ref. [0]. Therefore, an account of the "singular" part of the third-order correlation function and subsequent extraction of the "singular" part in the pairwise correlators leads us to the results equivalent to those obtained in terms of the Smoluchowski approach.

Hence, Eq.(12), in which one sets $g_{A B}(|\lambda|=R, t)=0$ and $K_{S}(d)=\infty$ reduces to the formal kinetic "law of mass action", while setting $g_{A B}(|\lambda|=R, t)=0$ and using $K_{S}(d)$ as in Eqs.(23) to (25), one obtains the effective kinetic equation of the Smoluchowski-type approach. Below, we proceed to show that taking into account the time evolution of the pairwise correlations, i.e. the term $g_{A B}(|\lambda|=R, t)$, one arrives at completely different physical behavior as compared to the ones predicted by the formal kinetic and Smoluchowski approaches.

Finally, we obtain the following system of equations, obeyed by the "fluctuational" part of the pairwise correlation functions. It reads

$$
\begin{gathered}
\dot{g}_{A B}(\lambda, t)=-K C(t)\left[2 g_{A B}(\lambda, t)+g_{A A}(\lambda, t)+g_{B B}(\lambda, t)\right]+ \\
+2 D \Delta_{\lambda} g_{A B}(\lambda, t)+I_{A B}(\lambda) \\
\dot{g}_{A A}(\lambda, t)=-2 K C(t)\left[g_{A B}(\lambda, t)+g_{A A}(\lambda, t)\right]+2 D \Delta_{\lambda} g_{A A}(\lambda, t)+I \delta(\lambda), \\
\dot{g}_{B B}(\lambda, t)=-2 K C(t)\left[g_{A B}(\lambda, t)+g_{B B}(\lambda, t)\right]+2 D \Delta_{\lambda} g_{B B}(\lambda, t)+I \delta(\lambda)
\end{gathered}
$$

Equations (26) to (28), accompanied by Eq.(22), are now closed with respect to mean particles' densities and pairwise correlations, and suffice the computation of the time evolution 
of the monomer-monomer reaction scheme.

\section{KINETICS OF THE MONOMER-MONOMER REACTION SCHEME.}

Below we will analyse solutions of Eqs.(22) to (28) in systems of different dimensionalities and with different types of external particle generation. The derivation of results in case of one-dimensional systems will be presented in detail. The steps involved for such a derivation in higher dimensions are essentially the same and here we will merely discuss the results.

\section{A. Low dimensional systems.}

Let us start with the case of one-dimensional systems in which an external source produces uncorrelated $A$ and $B$ particles.

We note first that the system of equations (26) to (28) possesses two integrable combinations

$$
S_{-}(\lambda, t)=2 g_{A B}(\lambda, t)-g_{A A}(\lambda, t)-g_{B B}(\lambda, t),
$$

which is related to the conserved property $Z(r, t)$, and

$$
S_{+}(\lambda, t)=2 g_{A B}(\lambda, t)+g_{A A}(\lambda, t)+g_{B B}(\lambda, t)
$$

These integrable combinations obey

$$
\dot{S}_{-}(\lambda, t)=2 D \Delta_{\lambda} S_{-}(\lambda, t)-2 I \delta(\lambda)
$$

which is thus the pure diffusive mode of the system, not affected by the reaction, and

$$
\dot{S}_{+}(\lambda, t)=2 D \Delta_{\lambda} S_{+}(\lambda, t)-4 K C(t) S_{+}(\lambda, t)+2 I \delta(\lambda)
$$

The desired property, i.e. the correlation function $g_{A B}(\lambda, t)$ which enters the Eq.(22), may be then expressed in terms of these integrable combinations as

$$
g_{A B}(\lambda, t)=\frac{1}{4}\left(S_{-}(\lambda, t)+S_{+}(\lambda, t)\right)
$$


Consider now the solutions to Eqs.(31) and (32) in one-dimensional systems. Applying the Fourier transformation over the variable $\lambda$,

$$
S_{ \pm}(\omega, t)=\frac{1}{\sqrt{2 \pi}} \int_{-\infty}^{\infty} d \lambda \exp (i \omega \lambda) S_{ \pm}(\lambda, t)
$$

to Eqs.(31) and (32), and assuming that at $t=0$ no $A$ and $B$ particles were present in the system, one readily gets that the Fourier-images of the integrable combinations follow

$$
\begin{aligned}
S_{-}(\omega, t) & =-I \sqrt{\frac{2}{\pi}} \int_{0}^{t} d \tau \exp \left(-2 D \tau \omega^{2}\right)= \\
& =-\frac{I}{D \omega^{2} \sqrt{2 \pi}}\left(1-\exp \left(-2 D \tau \omega^{2}\right)\right),
\end{aligned}
$$

and

$$
S_{+}(\omega, t)=-I \sqrt{\frac{2}{\pi}} \int_{0}^{t} d \tau \exp \left(-2 D \tau \omega^{2}-4 K \int_{\tau}^{t} d \tau^{\prime} C\left(\tau^{\prime}\right)\right)
$$

Now we notice that in the extreme situation, when reaction act occurs at any encounter of any $A$ and $B$ particle (i.e. when $K=\infty$ ) the second integrable combination $S_{+}(\omega, t)$ vanishes since the integral $\int_{\tau}^{t} d \tau^{\prime} C\left(\tau^{\prime}\right)$ is obviously positively defined. One can show, however, that even for the finite $K \mathrm{~s}$ the influence of $S_{+}(\omega, t)$ on the pairwise correlation function, Eq.(33), is not essential at large times and the dominant contribution to $g_{A B}$ comes from $S_{-}(\omega, t)$.

We note that setting $t=\infty$ in Eq.(35) we obtain that $S_{-}(\omega, \infty)$ has a steady-state spectrum of the form $S_{-}(\omega, \infty) \sim 1 / \omega^{2}$, i.e. the spectrum which has an essential singularity when $\omega \rightarrow 0$. Such a singular behavior of the fluctuation spectrum of the pairwise correlations in systems with binary reactions followed by an external uncorrelated production of the reactive species has been first obtained, in terms of a different than ours approach, in Refs. [16] and [17. The authors concluded thus that the steady state of such a system is highly anomalous; since such a singularity is not integrable in low dimensional systems, the steady state values of the integrable combination $S_{-}(\lambda, t)$ and thus of the correlation function $g_{A B}$ are infinitely large, which means that as time evolves the system progressively coarses into the domains containing particles of only one type.

Consider now how the integrable combination $S_{-}(\lambda, t)$ and the correlation function $g_{A B}$ grow in time. Taking the inverse Fourier transformation of the first line in Eq.(35) we get 


$$
S_{-}(\omega, t)=-\frac{I}{\sqrt{2 \pi D}} \int_{0}^{t} \frac{d \tau}{\sqrt{\tau}} \exp \left(-\lambda^{2} / 8 D \tau\right)
$$

The integrand in Eq.(37) is a bell-shaped function with its maximum at point $\tau=\lambda^{2} / 8 D$. For bounded $\lambda$, the bulk contribution to the integral comes from the algebraic tail $1 / \sqrt{\tau}$ and consequently, the leading at $t \gg R^{2} / 8 D$ behavior of the integrable combination follows

$$
S_{-}(|\lambda|=R, t)=-I \sqrt{\frac{2 t}{\pi D}}
$$

Accordingly, the absolute value of the "fluctuational" part of the pairwise correlation function grows in time as

$$
g_{A B}(|\lambda|=R, t)=-I \sqrt{\frac{t}{8 \pi D}}
$$

Inserting the just derived expression into the Eq.(22) and noticing that the correct asymptotical behavior of the mean density obtains when the terms in brackets compensate each other, i.e., when

$$
C(t)=\sqrt{-g_{A B}(|\lambda|=R, t)},
$$

we find that

$$
C(t)=I^{1 / 2}\left(\frac{t}{8 \pi D}\right)^{1 / 4},
$$

i.e. in one-dimensional systems with random uncorrelated generation of the reactive species the mean particle density grows sublinearly in time as time progresses. The behavior as in Eq.(41) has been also obtained earlier in Refs. [17 22].

Consider now how the situation will be changed in the case II, when $A$ and $B$ particles are introduced into the reactive bath as correlated pairs. In this case one readily gets that the Fourier-image of the integrable combination $S_{-}(\lambda, t)$ obeys the following equation

$$
\dot{S}_{-}(\omega, t)=-2 D \omega^{2} S_{-}(\omega, t)-2 I\left(1-\cos \left(\omega \lambda_{g}\right)\right)
$$

whose solution will read 


$$
S_{-}(\omega, t)=-\frac{I\left(1-\cos \left(\omega \lambda_{g}\right)\right)}{D \omega^{2} \sqrt{2 \pi}}\left(1-\exp \left(-2 D t \omega^{2}\right)\right)
$$

One may readily notice a very important feature of Eq.(43); in a striking contrast to the case I, here the steady-state spectrum is not singular in the limit $\omega \rightarrow 0$, but tends to a constant value

$$
S_{-}(\omega \rightarrow 0, t=\infty)=-\frac{I \lambda_{g}^{2}}{\sqrt{8 \pi} D},
$$

which means that $S_{-}(\lambda, \infty)$ and hence, $g_{A B}(\lambda, \infty)$ are bounded in systems of any dimensionality, and thus the well-defined steady-state mean density $C(t=\infty)$ also exists. We notice, however, that the steady-state pairwise correlation function is proportional to $\lambda_{g}^{2}$ and thus may increase indefinitely with growth of $\lambda_{g}$. This unbounded growth is, of course, quite consistent with the result in Eq.(39), since the limit $\lambda_{g} \rightarrow \infty$ corresponds to the case of uncorrelated generation of particles.

Now, the inverse Fourier transformation gives us

$$
\begin{aligned}
S_{-}(\lambda, t) & =-\frac{I}{\sqrt{2 \pi D}} \int_{0}^{t} \frac{d \tau}{\sqrt{\tau}}\left\{\exp \left(-\frac{\lambda^{2}}{8 D \tau}\right)-\right. \\
& \left.-\frac{1}{2} \exp \left(-\frac{\left(\lambda-\lambda_{g}\right)^{2}}{8 D \tau}\right)-\frac{1}{2} \exp \left(-\frac{\left(\lambda+\lambda_{g}\right)^{2}}{8 D \tau}\right)\right\}= \\
& =-\frac{I \lambda}{4 D \sqrt{\pi}}\left\{\Gamma\left(-1 / 2, \frac{\lambda^{2}}{8 D t}\right)-\frac{1}{2} \Gamma\left(-1 / 2, \frac{\left(\lambda_{g}-\lambda\right)^{2}}{8 D t}\right)-\right. \\
& \left.-\frac{1}{2} \Gamma\left(-1 / 2, \frac{\left(\lambda_{g}+\lambda\right)^{2}}{8 D t}\right)\right\},
\end{aligned}
$$

where $\Gamma(\alpha, x)$ denotes the incomplete Gamma function [51].

Consider now the asymptotic behavior of the pairwise correlation function, Eq.(33), for different values of parameters $\lambda$ and $\lambda_{g}$, and different values of time $t$.

One readily gets from Eq.(45) that at sufficiently short times, when $\lambda \gg \lambda_{g} \gg 8 D t$, the pairwise correlation function obeys

$$
g_{A B}\left(\lambda, \lambda_{g}, t\right)=-\frac{3 I \lambda_{g}^{2}(8 D t)^{3 / 2}}{8 \sqrt{\pi} D \lambda^{4}} \exp \left(-\frac{\lambda^{2}}{8 D t}\right),
$$

which shows that correlations drop off as a Gaussian function at large scales. 
Now, at short scales, such that $\lambda \ll \lambda_{g}$ and $\lambda \ll 8 D t$, and when $\lambda_{g}$ is sufficiently large, $\lambda_{g} \gg 8 D t$, we obtain that $g_{A B}\left(\lambda, \lambda_{g}, t\right)$ obeys Eq.(39), which is not a surprising result since at such scales the correlations in the particles' injection should be irrelevant.

Within the opposite limit, when $8 D t \ll \lambda \ll \lambda_{g}$ the correlator follows

$$
g_{A B}\left(\lambda, \lambda_{g}, t\right) \approx-\frac{I(8 D t)^{3 / 2}}{16 \sqrt{\pi} D \lambda^{2}} \exp \left(-\frac{\lambda^{2}}{8 D t}\right)
$$

which is reminiscent of the behavior in Eq.(46).

Finally, in the limit when both $\lambda \ll 8 D t$ and $\lambda_{g} \ll 8 D t$ (and $\lambda_{g}>\lambda$ ), i.e. in the limit of very long times, we find the following asymptotic expansion

$$
g_{A B}\left(\lambda, \lambda_{g}, t\right) \approx-\frac{I\left(\lambda_{g}-\lambda\right)}{16 D}\left[1-\frac{\lambda_{g}^{2}}{\lambda \sqrt{\pi D t}} \exp \left(-\frac{\lambda^{2}}{8 D t}\right)+\mathcal{O}(1 / t)\right], \lambda_{g} \geq \lambda
$$

where the symbol $\mathcal{O}(1 / t)$ signifies that the correction terms decay with time as $1 / t$.

Equation (48) suffices to derive the large- $t$ evolution of the mean particle density in the case of generation by correlated pairs, which reads

$$
C(t) \approx \sqrt{\frac{I\left(\lambda_{g}-R\right)}{16 D}}\left[1-\frac{\lambda_{g}^{2}}{2 R \sqrt{\pi D t}}+\mathcal{O}(1 / t)\right], \lambda_{g} \geq R
$$

Equations (49) reveals two surprising features; first, the steady-state density turns out to be dependent both on the diffusion constant and on the radius of pairs, generated by the source. Such a dependence is, of course, inconsistent with the predictions of the formal kinetic approach, based on the text-book "law of mass action". Second, the approach of particles' densities to their steady-state values obeys a power-law dependence, in a striking contrast to the exponential one predicted both by the formal kinetic and the Smoluchowski approach.

To close this subsection let us briefly consider the behavior of solutions of the reactiondiffusion equations (22), (26) to (28) in two-dimensional systems.

In the case I, we have from Eqs.(31) and (33) that as $t \rightarrow \infty$ the pairwise correlation function grows (by absolute value) as

$$
g_{A B}(|\lambda|=R, t) \approx-I \ln \left(D t / R^{2}\right), D t \gg R^{2},
$$


and consequently, we get from Eq.(40) that in this case at large times the mean particle density exhibits logarithmically slow growth [16 22],

$$
C(t) \approx \sqrt{I \ln \left(D t / R^{2}\right)}
$$

Now, in the case II, we obtain that the steady-state exists and $g_{A B}(|\lambda|=R, \infty)$ behaves as

$$
g_{A B}(|\lambda|=R, \infty) \approx-I \ln \left(\lambda_{g}\right),
$$

and hence, the steady-state density turns to be a slowly growing function of the radius of the generated pairs,

$$
C(t=\infty) \approx \sqrt{I \ln \left(\lambda_{g}\right)}
$$

Some analysis shows also that such a steady-state is approached via an algebraic law

$$
C(t)-C(t=\infty) \approx(D t)^{-1}
$$

in contrast to the exponential in time dependence predicted by mean-field descriptions.

\section{B. Three-dimensional systems.}

As we have already mentioned, in the case I the steady-state fluctuation spectrum is characterized by an essential singularity of the type $1 / \omega^{2}$ as $\omega \rightarrow 0$. In three-dimensional systems such a singularity is integrable, which insures that the steady-state correlations exist and vanish as $\lambda \rightarrow \infty$. Consequently, the steady-state mean particle density $C(t=\infty)$ should exist in 3D also in this case. Let us analyse now the form of these correlations. Solving Eq.(31) in 3D we find (up to the correction terms which are exponentially small with $K$ ) that

$$
g_{A B}(\lambda, t=\infty) \approx-\frac{I}{8 \pi D \lambda}
$$

i.e. $A-B$ correlations vanish with the distance between particles $\lambda$ as $1 / \lambda$, which shows that in the monomer-monomer catalytic scheme taking place in three dimensional systems the 
correlations in the steady-state show a quasi-long-range order decaying as the first inverse power of the interparticle distance.

Now, substituting Eq.(55) into the Eq.(22) we find the following expression for the steadystate density in 3D,

$$
C(t=\infty)=\sqrt{\left(\frac{1}{8 \pi D R}+\frac{1}{K}\right) I},
$$

which shows that $A-B$ correlations lead here to an effective renormalization of the reaction constant in the steady-state, i.e. $C(t=\infty)$ has the form $C(t=\infty)=\sqrt{I / K_{a p p}}$, where $K_{a p p}$ is the mentioned above apparent reaction constant [7].

Consider now how such a steady-state is approached at long times. Expanding the solution of Eq.(31) near the steady-state, we have that pairwise correlations approach the steady-state as a power-law,

$$
g_{A B}(|\lambda|=R, t) \approx \frac{I}{8 \pi D R}\left[1-\frac{R}{\sqrt{\pi D t}}+O(1 / t)\right]
$$

which yields, in turn, a power-law relaxation of the mean particle density to the steady-state

$$
C(t)-C(t=\infty) \approx(D t)^{-1 / 2}
$$

Therefore, in contrast to low dimensional systems, in three dimensional systems with random uncorrelated generation of the reactive species the essential singularity in the fluctuation spectrum is integrable, correlations vanish with the distance between particles and the steady-state mean particle density exists. However, the steady-state density is different from that predicted by the mean-field "law of mass action" and shows, in particular, dependence on the particles' diffusivity D. Besides, Eq.(58) reveals that here approach to the steady-state is described by a power-law with the characteristic exponent $-1 / 2$, which is essentially non-mean-field behavior.

Finally, for the case II we find the following results for the correlation function and mean density. In the steady-state the $A-B$ correlations are equal to zero for $\lambda \geq \lambda_{g}$ (again, apart from the exponentially small with $K$ terms). In the domain $\lambda<\lambda_{g}$ the correlations exist and are described by 


$$
g_{A B}(\lambda, t=\infty) \approx-\frac{I}{8 \pi D \lambda}\left(1-\frac{\lambda}{\lambda_{g}}\right),
$$

which reduces to the result in Eq.(55) when $\lambda_{g}=\infty$. In constrast to the behavior as in Eq.(55), however, the correlations vanish at finite values of the correlation parameter $\lambda$.

Now, Eq.(59) yields for the steady-state mean-particle density

$$
C(t=\infty)=\sqrt{I\left(\frac{1}{K}+\frac{1-R / \lambda_{g}}{8 \pi D R}\right)},
$$

which is less than the steady-state density in the case I, Eq.(56), due to a factor $1-R / \lambda_{g}$,

which renormalizes the Smoluchowski constant. Consequently, for $\lambda_{g}>R$ apparent rate constant here takes the form

$$
K_{a p p}=\frac{8 \pi D R K}{8 \pi D R+\left(1-R / \lambda_{g}\right) K}
$$

We find also that such a steady-state is approached via a power-law,

$$
C(t)-C(t=\infty) \approx(D t)^{-3 / 2}
$$

which is faster than the approach described in Eq.(58), but still very different from the exponential behavior predicted by mean-field analysis.

\section{CONCLUSION.}

To summarize, we have shown that both the cases I and II the fluctuations effects dominate the kinetics of the monomer-monomer catalytic scheme involving diffusive particles and induce essential departures from the predictions of the mean-field approaches. In the case I, the effects of fluctuation are especially pronounced in low dimensional systems - the steady-state does not exist and mean particle density grow indefinitely in time, in absence of hard-core exclusion between particles. In three dimensions the steady-state exists, but is characterized by very strong interparticle correlations, which, in turn, have a strong impact on the value of the steady-state mean particle density. The steady-state density is different from that predicted by mean-field "law of mass action". The approach to this steady-state 
is described by an anomalous power-law with the characteristic exponent $-1 / 2$, which stems from the presence of essential singularity in the steady-state fluctuation spectrum. In the case II, the steady-state fluctuation spectrum and the steady-state mean particle density exist in any dimension, but show an anomalous, non-mean-fields dependence on the particles' diffusivity and the radius of pairs, generated by the source. Approach to the steady-state follows a universal power-law with the characteristic exponent $-d / 2$, which resembles, apart from the dependence of the prefactors on the system parameters (e.g. constant of the backward reaction) the long-time approach to the equilibrium in reversible chemical reactions 12, $13,38,42$. The origin of such a behavior is that the fluctuation spectrum in the steadystate is flat at small values of the wave-vector, i.e. the essential singularity in the steady-state spectrum of fluctuations is screened.

[1] R.Eiswirth, K.Krischer and G.Ertl, Appl. Phys. A 51, 79 (1990)

[2] A.Zangwill, Physics at Surface, (Cambridge University Press, Cambridge, 1990) pp 400-420

[3] D.Avnir, R.Gutfriend and D.Farin, Fractals in Science, A.Bunde and S.Havlin, eds., (Springer, Berlin, 1994)

[4] A.Osaki and K.Aika, Catalysis Science and Technology 1 (Springer, Berlin, 1981) pp 87-167

[5] D.W.Goodman, Appl. Surf. Science 19, 1 (1984)

[6] M. von Smoluchowski, Z. Phys. B 16, 321 (1915)

[7] F.Collins and G.Kimball, J. Colloid Sci. 4, 425 (1949)

[8] S.A.Rice, Diffusion-Limited Reactions, in: Chemical Kinetics 25, C.H.Bamford, C.F.H.Tipper and R.G.Compton, eds., (Elsevier, Amsterdam, 1985)

[9] A.A.Ovchinnikov, S.F.Timashev and A.A.Belyy, Kinetics of Diffusion Controlled Chemical 
Processes (Nova Science, New York, 1989)

[10] A.A.Ovchinnikov and Ya.B.Zeldovich, Chem. Phys. 28, 214 (1978)

[11] S.F.Burlatsky, Theor. Exp. Chem. 14, 483 (1978)

[12] Ya.B.Zeldovich and A.A.Ovchinnikov, Sov. Phys. JETP Lett. 26, 440 (1977)

[13] Ya.B.Zeldovich and A.A.Ovchinnikov, Sov. Phys. JETP 47, 829 (1978)

[14] D.Toussaint and F.Wilczek, J. Chem. Phys. 78, 2642 (1983)

[15] S.Redner and K.Kang, Phys. Rev. Lett. 51, 1729 (1983)

[16] A.A.Ovchinnikov and S.F.Burlatsky, Sov. Phys. JETP Lett. 43, 638 (1986)

[17] S.F.Burlatsky, A.A.Ovchinnikov and K.A.Pronin, Sov. Phys. JETP 65, 353 (1987)

[18] R.Kopelman, Science 241, 1620 (1988)

[19] Y.C.Zhang, Phys. Rev. Lett. 59, 1726 (1987)

[20] D.ben-Avraham and C.R.Doering, Phys. Rev. A 37, 5007 (1988)

[21] E.Clément, L.Sander and R.Kopelman, Phys. Rev. A 39, 6472 (1989)

[22] G.Oshanin, S.F.Burlatsky and A.A.Ovchinnikov, Phys. Lett. A 139, 245 (1989)

[23] A.Blumen, J.Klafter and G.Zumofen, in: Optical Spectrocopy of Glasses, I.Zschokke, ed., (Reidel Publ.Co, Dordrecht, 1986)

[24] G.Oshanin, M.Moreau and S.F.Burlatsky, Adv. Colloid and Interface Sci. 49, 1 (1994)

[25] G.Oshanin, I.M.Sokolov, P.Argirakis and A.Blumen, J. Chem. Phys. 105, 6304 (1996)

[26] R.Ziff, E.Gulari and Y.Barshad, Phys. Rev. Lett. 56, 2553 (1986)

[27] K.Fichthorn, E.Gulari and R.Ziff, Phys. Rev. Lett. 63, 1527 (1989)

[28] E.Clément, P.Leroux-Hugon and L.Sander, Phys. Rev. Lett 67, 1661 (1991) 
[29] S.Redner and H.Takayasu, J. Phys. A 63, L4207 (1990)

[30] C.Flament, E.Clément, P.Leroux-Hugon and L.Sander, J. Phys. A 25, L1317 (1992)

[31] K.Fichthorn, E.Gulari and R.Ziff, Chem. Eng. Sci. 44, 1403 (1989)

[32] E.Clément, R.Kopelman and L.Sander, J. Stat. Phys. 65, 919 (1991)

[33] A.Malevanets, A.Careta and R.Kapral, Phys. Rev. E 52, 4724 (1995)

[34] J.Klafter, G.Zumofen and A.Blumen, J. Phys. A 24, 4835 (1991)

[35] E.V.Albano, Phys. Rev. Lett. 69, 656 (1992); Surf. Sci. 235, 351 (1990).

[36] A.Casties, J.Mai and W. von Niessen, J. Chem. Phys. 99, 3082 (1993)

[37] Y.E.L. Koo and R.Kopelman, J. Stat. Phys. 65, 893 (1991)

[38] G.Oshanin and S.F.Burlatsky, J. Phys. A 22, 973; 977 (1989)

[39] S.F.Burlatsky, A.A.Ovchinnikov and G.Oshanin, Sov. Phys. JETP 68, 1153 (1989)

[40] S.F.Burlatsky, G.Oshanin and A.A.Ovchinnikov, Chem. Phys. 152, 13 (1991)

[41] S.F.Burlatsky and G.Oshanin, J. Stat. Phys. 65, 1095 (1991)

[42] G.Oshanin, A.Mogutov, M.Moreau and S.F.Burlatsky, J. Mol. Liquids 63, 175 (1995)

[43] S.F.Burlatsky and A.A.Ovchinnikov, Russ. J. Phys. Chem. 54, 1741 (1980) (the English translation of this paper can be also found in [9], Sec.1.7)

[44] S.F.Burlatsky and M.Moreau, Phys. Rev. E 51, 2363 (1995)

[45] G.Oshanin, A.Stemmer, S.Luding and A.Blumen, Phys. Rev. E 52, 5800 (1995)

[46] G.Oshanin and A.Blumen, J. Chem. Phys. 108, 1140 (1998)

[47] G.Oshanin, A.Chernoutsan and S.F.Burlatsky, Theor. Exp. Chem. 26, 12 (1990)

[48] T.R.Waite, Phys. Rev. 107, 463 (1957) 
[49] D.C.Torney and H.M.McConnell, Proc. R. Soc. Lond. A 387, 147 (1983); J. Phys. Chem. 87, $1941(1983)$

[50] V.Kuzovkov and E.Kotomin, Rep. Prog. Phys. 51, 1479 (1988)

[51] Handbook of Mathematical Functions, eds.: M.Abramowitz and I.A.Stegun (Dover Publ., New York, 1972)

\section{Figure Caption.}

Fig.1. Langmuir-Hinshelwood reaction on a two-dimensional catalytic substrate. Black

and grey spheres denote particles of $A$ and $B$ species, respectively; (1) describes the situation in which an $A$ and a $B$ appear within the reactive distance from each other and may enter into reaction. 


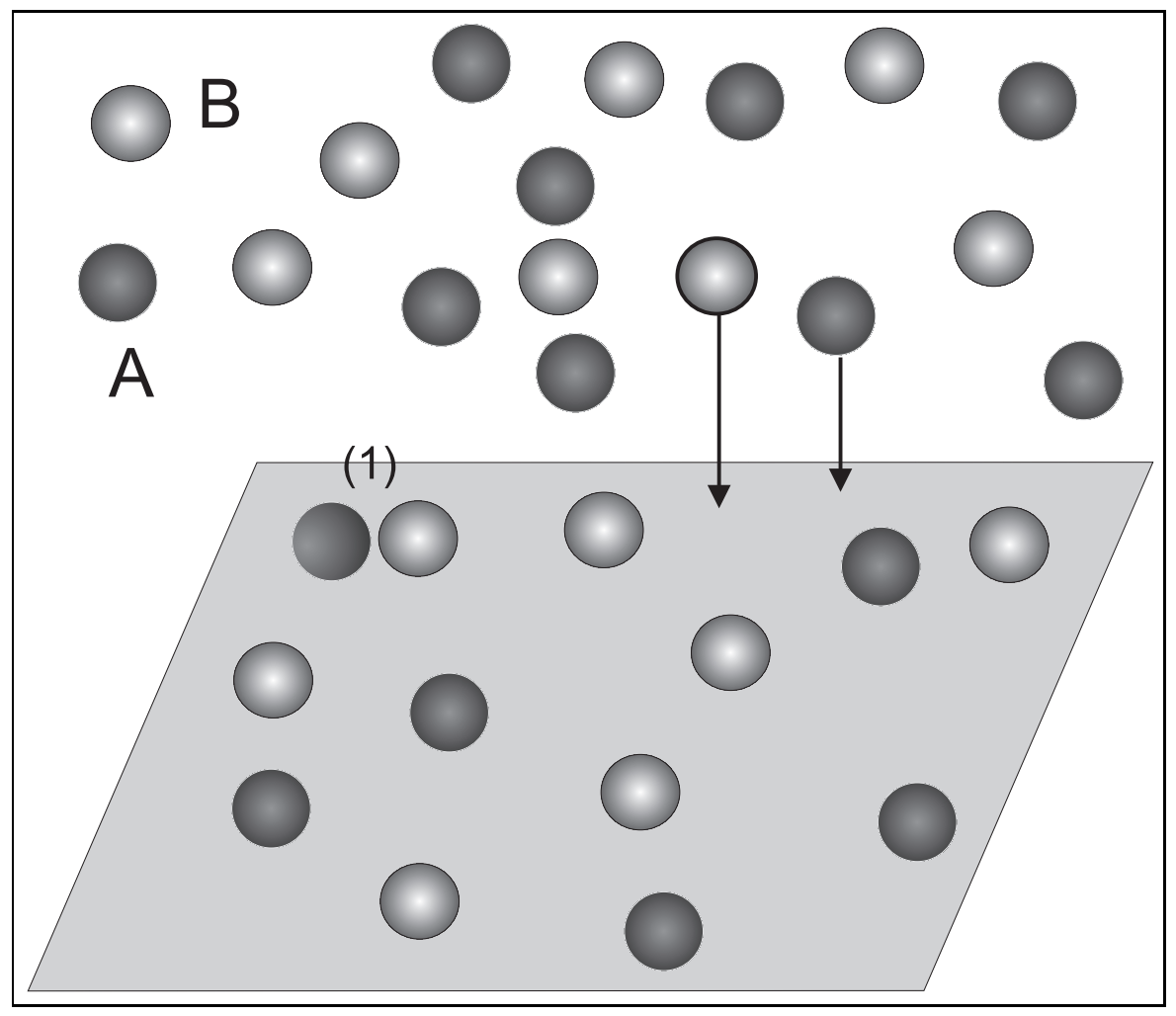

Fig. 1. Argyrakis et al., J. Chem. Phys. 